\title{
GUIA DE MANEJO Y LÍNEA DE INVESTIGACIÓN EN PACIENTES CON LABIO Y|O PALADAR HENDIDO, EN LA UNIVERSIDAD SANTO TOMÁS EN BUCARAMANGA
}

\author{
Ethman Ariel Torres Murillo \\ Odontólogo, U. Santo Tomás, Estomatólogo Pediatra, U. Nacional de Colombia, Ortodoncista, \\ U. Santo Tomás, Docente, U. Santo Tomás
}

Autor responsable de la correspondencia: Ethman Ariel Torres $M$.

Correo electrónico: ethmant@yahoo.com

\begin{abstract}
Presentación realizada en el II Simposio de Actualización en Odontología Pediátrica en Bogotá, Noviembre de 2005.
\section{RESUMEN}

La universidad Santo Tomás, seccional Bucaramanga cuenta con una clínica destinada a la atención de pacientes con labio y/o paladar hendido. Se ofrece un servicio integral para atender a los pacientes del nororiente colombiano. Por esta razón, también se ha creado un grupo que investiga algunos aspectos relacionados con esta malformación para entender un poco más su causalidad, manejo y secuelas, y así ofrecer un tratamiento oportuno al paciente y a sus familias. El presente trabajo presenta un resumen de la guía de manejo utilizado en estos pacientes y la línea de investigación desarrollada en la Universidad en esta área. [Torres EA. Guía de manejo y línea de investigación en pacientes con labio y/o paladar hendido, en la Universidad Santo Tomás en Bucaramanga. Ustasalud Odontología 2005; 4: 109 - 115]
\end{abstract}

Palabras clave: Labio y/o paladar hendido, Guía de manejo.

\section{MANAGEMENT GUIDELINES AND RESEARCH IN PATIENTS WITH CLEFT LIP/PALATE, IN THE SANTO TOMAS UNIVERSITY OF BUCARAMANGA}

\begin{abstract}
The Santo Tomas University in Bucaramanga, counts with a clinic destined to the attention of patients with cleft lip/palate. An integral service is offered to take care of these patients from the Colombian noreast. Therefore, also a group has been created that investigates some aspects related to this malformation to understand a little more about its causality, handling and sequels, and thus to offer an opportune treatment to the patients and their families. The present work presents the management guidelines used in these patients and the research developed in the University in this area.
\end{abstract}

Key words: Cleft lip/palate, Management guidelines.

Recibido para publicación: 31 de octubre de 2005. Aceptado para publicación: 12 de diciembre de 2005.

\section{INTRODUCCIÓN}

La universidad Santo Tomás, seccional Bucaramanga cuenta con una clínica destinada a la atención de pacientes con labio y/o paladar hendido. La clínica ofrece un servicio integral en las áreas de: odontopediatría, psicología, fonoaudiologia, cirugía maxilofacial, genética, ortodoncia y odontología general a pacientes del nororiente colombiano, remitidos de centros hospitalarios de la región.
El grupo surge ante la falta de una entidad que maneje los pacientes de manera integral en esta región del país. Como se sabe, los niños que nacen con labio y/o paladar hendido requieren de la colaboración de numerosos especialistas para garantizar la atención apropiada de todos los aspectos que se relacionan con su patología. Dentro de este equipo integral, el odontólogo pediatra y el ortodoncista son parte fundamental en todas las etapas de desarrollo del paciente, como integrante y líder de un 
equipo, que vela por ofrecer al paciente un servicio de alta calidad y, a la vez por crear investigación en esta área.

El presente trabajo presenta un resumen del protocolo de manejo utilizado en estos pacientes y la línea de investigación desarrollada en la Universidad en esta área.

\section{GUIA DE MANEJO}

A continuación se exponen los procedimientos odontológicos realizados en las clínicas odontológicas de la Universidad a los pacientes con labio y/o paladar hendido. Por convención, el tratamiento se divide en fases según la edad del paciente y obedece a las guías de manejo de la institución. Se debe tener en cuenta que se expone el tratamiento ideal a realizar, ya que la mayoría de las veces los pacientes no se atienden secuencialmente y es dificil sean revisados por todos el equipo interdisciplinario.

\section{FASE I: NACIMIENTO - 18 MESES}

El manejo del paciente se inicia con la atención inmediata a las necesidades del recién nacido; en estos niños se observan trastornos de alimentación, que dificultan una nutrición adecuada, por lo que parte de la labor es enseñar a la madre como alimentar a su bebe y realizar una higiene adecuada en la hendidura. La mayoría de nuestros pacientes se inicia con manejo de técnicas de lactancia de materna y remisión a los demás integrantes del grupo interdisciplinario. Se cuenta con la ayuda del área de odontopediatría de la Universidad.

El tratamiento ortopédico en esta edad continua siendo controversial al igual que el uso de placas obturadoras. En la literatura se encuentran numerosos reportes de utilización de ortopedia prequirúrgica en estas edades. Desde 1950, McNeil sugirió el uso de aparatos ortodónticos en el tratamiento inicial prequirúrgico de niños con labio y/o paladar hendido con el objeto de guiar los segmentos separados a una correcta configuración del arco. ${ }^{1,2}$ Actualmente, se considera que estos métodos ofrecen menos ventajas a largo plazo de lo que se pensaba ${ }^{3}$ por lo que no son empleados por muchos centros de atención interdisciplinaria. De igual modo, se han utilizado injertos óseos en estas edades ${ }^{4} \mathrm{y}$, actualmente, existe el convencimiento casi unánime de que los injertos primarios del proceso alveolar están contraindicados ya que tienden a interferir con el crecimiento posterior. ${ }^{5}$ Aunque los que defienden esta técnica aseguran que al utilizarlos previenen el colapso de la zona anterior y permiten la erupción adecuada de todos los dientes; sin embargo, los resultados muestran que son más los efectos adversos que sus ventajas, razón por lo que no se manejan en la clínica. Esta primera fase se constituye en el primer acercamiento del grupo con el paciente y su familia, se debe procurar despejar todos los temores y brindar confianza y asesoría en todas las dudas de los padres.

\section{FASE II: FASE DE DENTICIÓN TEMPORAL}

El tratamiento durante esta fase del desarrollo dental tiene como objetivo fundamental el establecer y mantener una adecuada salud bucal, se debe manejar una meticulosa higiene oral diaria e insistir en el papel de los padres en este campo. Se realizan visitas cada tres 0 cuatro meses que permitan al odontólogo interceptar los signos de daño; este régimen preventivo se mantiene durante todas las fases de manejo del paciente y debe ser objetivo común de todos los integrantes del grupo. ${ }^{6} \mathrm{El}$ esquema de prevención ha de ser individual en cada paciente, según su susceptibilidad y daño de las estructuras dentarias. Es necesario tener en cuenta que es frecuente la presencia de alteraciones en la estructura del esmalte como las hipoplasias e hipocalcificaciones.? Otro aspecto a considerar es la presencia de dientes supernumerarios. ${ }^{8}$ Se deben extraer cuando ocasionan apiñamiento 0 interferencias con la erupción dental normal aunque, a veces, se puede considerar la posibilidad de no extraerlos para conservar el hueso alveolar. ${ }^{9}$

Varios de los problemas de oclusión que presentan los niños con hendiduras palatinas en la edad de dentición temporal no se deben a la propia hendidura, sino a los efectos de las técnicas quirúrgicas, que aunque han mejorado, afectan el crecimiento craneofacial. El cierre del labio deja inevitablemente alguna constricción en la parte anterior del arco superior y el cierre del paladar provoca algún grado de constricción lateral. ${ }^{10}$ Por lo tanto, estos pacientes tienden a una mordida cruzada anterior y posterior, cosa que no sucede en los no tratados. ${ }^{11}$ Sin embargo, estos procedimientos deben realizarse por razones funcionales y por el desarrollo del lenguaje, de tal modo que, el tratamiento de ortodoncia en estos pacientes es una parte necesaria de la rehabilitación integral. En nuestro grupo, además de manejar la parte de prevención, el paciente es valorado conjuntamente por 
el ortodoncista y el odontólogo pediatra para realizar el diagnóstico y la valoración de cada caso en particular y así establecer sus prioridades y objetivos de tratamiento.

El ortodoncista debe valorar y realizar exámenes periódicos que permitan identificar anormalidades y tratarlas de manera oportuna. Ante la presencia de discrepancias transversales o sagitales se cuenta con aparatología tanto fija como removible (placas de expansión, quadhelix o máscara facial). ${ }^{12}$ El objetivo es mantener el paciente en unas relaciones intra e interarco adecuadas que permitan un crecimiento normal. El paciente debe ser valorado como integrante de una familia teniendo en cuenta pautas de manejo del comportamiento según cada caso individual.

\section{FASE III: FASE DE DENTICIÓN MIXTA}

Muchos de los problemás que surgen al ortodoncista durante esta fase de desarrollo dental se originan con la erupción ectopica de los incisivos permanentes centrales y laterales, o en las mordidas cruzadas de los segmentos posteriores 0 anteriores. Los problemás que más se presentan en esta edad son: mordidas cruzadas posteriores, incisivos permanentes mal alineados, discrepancias anteroposteriores, anomalías dentales y discrepancias verticales. ${ }^{13}$

Para corregir las mordidas cruzadas posteriores se realiza una expansión; hay que tener en cuenta que no existe una sutura palatina media y que la cicatriz después de la palatorrafia puede agravar el colapso del arco. ${ }^{14}$ Además, se debe considerar que en la dentición permanente, probablemente, se necesitara una reexpansión ya que el crecimiento continua. En relación con esta situación, en la clínica se utilizan aparatos fijos o removibles según las necesidades y el estudio diagnóstico de cada paciente. Para corregir los incisivos mal alineados se puede utilizar aparatología fija con brackets en los dientes anteriores estos dientes pueden producir daño al labio y estar predispuestos a la fractura, además de la parte estética y funcional del paciente. Para tratamiento de discrepancias anteroposteriores el tratamiento de elección es la máscara facial. ${ }^{15,16}$ Esta dirige la fuerza extraoral hacia abajo y adelante en el área canina. El tratamiento se inicia según las necesidades del paciente pero es recomendable en la dentición decidua o mixta temprana.${ }^{16}$ Luego se manejaran periodos de retención que pueden llevarse a cabo con aparatos funcionales como el Frankell. ${ }^{17}$
Los injertos óseos se realizan con el objetivo de dar continuidad al maxilar, permitir la erupción dental, de tal modo que el injerto debe, al integrarse, proveer una vía de erupción para el canino. Adicionalmente, facilita los movimientos ortodonticos, ya que la falta de hueso no permite la aproximación de dientes adyacentes; el injerto al rellenar el defecto debe actuar como corredor óseo para permitir este movimiento. El injerto debe aportar soporte periodontal a los dientes vecinos. Debe cerrar la fistula oro-nasal remanente a nivel del vestíbulo oral. Debe brindar sustrato para la colocación de prótesis o implantes si son necesarios y proporcionar un soporte al ala nasal. En la institución se realizan injertos autologos y el sitio donante se selecciona de acuerdo con el volumen del defecto. Son realizados por el área de cirugía maxilofacial. ${ }^{18}$

En resumen, el ortodoncista y odontólogo pediatra deben valorar cuidadosamente a cada paciente y procurar mantener unas relaciones transversales y sagitales adecuadas durante todo este periodo, para ello cuentan con aparatología como las placas de expansión, quad helix, máscara facial, y aparatos funcionales como el Frankell. La utilización de uno u otro aparato es una decisión individual; idealmente, el paciente debe ser valorado anualmente por todo el grupo interdisciplinario fijando unos objetivos claros y precisos a cumplir.

\section{FASE IV: FASE DE DENTICIÓN PERMANENTE}

En la dentición permanente el tratamiento ortodontico se dirige a la corrección de dientes malalineados, corrección de discrepancias sagitales, horizontales y verticales, el establecimiento de unas relaciones oclusales optimás y un largo periodo de retención. ${ }^{19} \mathrm{El}$ ortodoncista ha de ser cuidadoso en el proceso diagnóstico con todas las ayudas necesarias y disponibles. El paciente antes de iniciar tratamiento, debe ser presentado en junta interdisciplinaria, donde en conjunto con el cirujano plástico, maxilofacial, ortodoncista, rehabilitador, periodoncista y demás integrantes del grupo se tome la mejor decisión para el paciente.

Algunos pacientes sólo necesitaran tratamiento ortodóntico correctivo; otros, ortodoncia prequirúrgica, más cirugía, y otros ortodoncia más rehabilitación. El ideal es que el paciente llegue a esta etapa de tratamiento con un mínimo de discrepancias, éste ha de ser el objetivo común de todo el grupo. Al pasar los años, se ha visto que cada vez son mejores los tratamientos que ofrecen todos 
los grupos a nivel mundial. En los años setenta, la mitad de los pacientes necesitaban prótesis fija y un 10\% 0 15\% necesitaban la cirugía ortognatica. A finales de los ochenta, Semb y colaboradores reportaron que menos del 10\% de sus pacientes requerían tratamiento protésico y rara vez realizaban cirugías ortognáticas en sus pacientes, si estos habían sido controlados todo el tiempo. ${ }^{20}$ Pero en algunos pacientes con hendidura, el crecimiento continúo de la mandíbula, tras finalizar el tratamiento ortodóncico, da lugar a recidivas de mordidas cruzadas anteriores y posteriores, posiblemente debido al menor crecimiento del maxilar superior anteroposterior 0 vertical. Por lo que la cirugía ortognatica puede ser la última fase en el tratamiento de estos pacientes.

\section{LÍNEA DE INVESTIGACIÓN}

\subsection{VARIABLES SOCIODEMOGRÁFICAS EN PACIENTES CON LABIO Y|O PALADAR HENDIDO ${ }^{21}$}

La fisura labio palatina (FLP) se encuentra dentro de las malformaciones congénitas más frecuentes en todo el mundo; aunque los estudios epidemiológicos y genéticos sobre esta anomalía son númerosos, su etiología se considera de origen multifactorial.

Son múltiples las causas de este defecto de nacimiento, incluyendo genes mutantes heredados y agentes teratogénicos. Se acepta en general que el factor etiológico principal de la FLP es de carácter genético, aunque se sugiere una génesis mixta, compuesta por causas ambientales y genéticas.

Esto manifiesta la necesidad de seguir investigando cuáles podrían ser los factores etiológicos causantes de la FLP, para avanzar en el desarrollo de nuevas herramientas en prevención, al tener más claro los factores involucrados con su desarrollo. El propósito de la presente investigación fue encontrar posibles asociaciones entre los factores del medio ambiente y la aparición de la fisura labio palatina.

Se tomo una muestra de conveniencia conformada por 195 individuos que asistieron a la convocatoria realizada por la organización Operación Sonrisa en las ciudades de Bogotá y Bucaramanga, que cumplían con los criterios de inclusión. La información fue recolectada en tres instrumentos. Al analizarlos se demostró que la FLP fue (74.8\%) más frecuente que la labial (25.13\%), con mayor incidencia en el lado izquierdo de la cara, en raza mestiza, genero masculino y población urbana de estratos bajos.

Se encontró que dentro de los factores de riesgo examinados, la exposición de la madre durante el primer trimestre de embarazo a la ingesta de medicamentos, exposición a fungicidas, enfermedades crónicas y el estrato socioeconómico bajo se asocia, pero no significativamente, con la aparición de la fisura labio palatina. Estos datos apoyan a la necesidad de un manejo preventivo futuro de dicha malformación.

\subsection{CIGARRILLO Y ALCOHOL COMO FACTOR DE RIESGO PARA LABIO Y|O PALADAR HENDIDO E HIPODONCIA ${ }^{22}$}

Los resultados de este estudio muestran que la hendidura labio palatina es la más frecuente; se presenta en 76 pacientes que corresponde al $96 \%$. Siendo el lado derecho el más afectado, en 47 pacientes (59\%); el izquierdo, en 21 pacientes (27\%) y bilateral, en 11 pacientes (14\%). Esto se relaciona con el lado en que se presenta la hipodoncia. Se presentó hipodoncia derecha en 47 pacientes (59\%), izquierda en 20 pacientes (25\%) y bilateral en 12 (16\%). Se encontró hipodoncia en 67 pacientes del grupo estudio (85\%). La hipodoncia del incisivo lateral superior fue la más frecuente, en 64 pacientes (81\%) y la hipodoncia de premolares en 5 pacientes $(7 \%)$.

Al comparar los resultados del estudio con los resultados de otros estudios, también se encuentra que la hendidura más frecuente es la labio palatina. Ericson y colaboradores en 1979, estudiaron un grupo de 68 pacientes, 51 presentaron labio y paladar. Khcury y colaboradores en 1989, en un grupo de 345 pacientes, 238 con labio y paladar hendido. Van den Eden (1990), estudió una muestra de 173 pacientes, 123 con labio y paladar hendido. Shaw y colaboradores en 1991, en un grupo de 731, 447 con labio y paladar hendido. ${ }^{23}$ Shapira y colaboradores (2000), reportó en un grupo de 238 pacientes, 198 con Labio y paladar hendido. ${ }^{24}$ En el presente estudio de 79 pacientes se encontraron 76 con labio y paladar hendido.

Los resultados sobre hipodoncia muestran que se presenta en alta frecuencia en pacientes con labio y paladar. El presente estudio muestra una frecuencia de hipodoncia del $85 \%$, siendo el $81 \%$ para hipodoncia de laterales y $7 \%$ 
para hipodoncias de premolares. Shapira encontró 74\% de hipodoncia de laterales y $18 \%$ de premolares. ${ }^{25}$ Yeyoshua en una muestra de 278 pacientes encontró hipodoncia en 213 pacientes (77\%) siendo el incisivo lateral el más frecuente, en el $74 \%$ de los casos..$^{25}$ lo que es concordante a nuestros resultados. Se reportaban cifras de hipodoncia de laterales en la población normal del $2.2 \%$ y en pacientes con labio y paladar del $56.9 \%$. En un estudio realizado en Colombia, se busco establecer el patrón de herencia de agenesias dentales en siete grupos familiares colombianos, encontrando que los dientes ausentes con mayor frecuencia fueron los laterales superiores (46.1\%) seguido por los segundos premolares inferiores (7.69\%). ${ }^{26}$ Los resultados del presente estudio muestra hipodoncia de premolares del 7\% en pacientes con labio y paladar hendido. Estos datos concuerdan con resultados publicados en pacientes con hendiduras, han sido del 3.4\% y del 6.6\%, aunque el estudio de Shapira encontró un $18 \%$ de hipodoncia de premolares, en un grupo con mayor porcentaje de labio y paladar hendido. También, se ha asociado la hipodoncia con gran cantidad de síndromes craneofaciales, dentro de los que tenemos la secuencia de Pierre Robin (69\%), sindrome de Van der Woude (70\%), diferentes formas de Displasia Ectodermica y otros síndromes craneofaciales relacionados con hendiduras labio-palatinas. ${ }^{24}$

La alta frecuencia de hipodoncia en pacientes con labio y paladar nos sugiere la relación entre mecanismos de formación de fisuras orales y procesos de odontogénesis. Los dos procesos dependen de la gobernabilidad de la migración de las células de la cresta neural y de interacciones epitelio mesenquima. Además, se ha mostrado que pueden tener la misma influencia genética. Satokata (1994) utilizó ratones transgénicos de MSX1 no funcional, género anodoncia y paladar hendido, mostró como el mismo grupo de genes que intervienen en la formación de la cara, están relacionados con la formación dental. Una de las explicaciones de este desorden facial, es que la expresión del MSX1 en las células mesenquimales derivadas de la cresta neural se haya en los procesos faciales y dentales. ${ }^{22}$ Thesleff en 1997, señaló que los genes homeóticos, con sus factores de transcripción y de crecimiento estan implicados en la regulación de la morfogénesis y en el desarrollo craneofacial y dental del ser humano; mostró como algunas proteínas como la proteína morfogénetica del hueso (BMP4), interviene en la regulación de la formación de estructuras esqueléticas, además de actuar como señal inductora epitelial en el desarrollo de los dientes, regulando la expresión génica en el mesenquima dental, que incluye la expresión del MSX1, importante para la iniciación de dichas estructuras. $^{25}$

Otro de los estudios que resalta la evidencia de la base genética para hendiduras orales e hipodoncia lo constituye las investigaciones de Vastardis y colaboradores, quienes localizaron la mutación del gen MSX1 en el cromosoma 4 (4p16) en todos los miembros afectados de una familia con hipodoncia de segundos premolares. ${ }^{23}$ En humanos, la inactivación de una copia de los genes causa defectos dentales o defectos dentales y hendiduras en el caso mutación MSX1. ${ }^{26}$

Estos datos son de interés ya que establece la asociación entre hendiduras orales e hipodoncias en relación con el labio y paladar hendido. El estudio muestra una relación directa entre el lado de la hendidura y el lado en que se presenta la hipodoncia; el 47\% de las hendiduras se presento en el lado derecho y el $47 \%$ de las hipodoncias de laterales se presento en el mismo lado. Lo que relaciona, a nivel biológico, la formación de la fisura con la presencia de la hipodoncia.

Según el estudio del árbol genealógico de la muestra, 39 pacientes (49\%) reportaron tener antecedes de labio y/o paladar hendido, mostrando un patrón poligénico por encontrarse la presencia de hendidura labio y/o palatina en varias líneas generacionales, tanto por herencia materna como paterna. Es importante resaltar que no se encuentra un patrón autosómico dominante o recesivo que pueda demostrar la intervención de factores ambientales como pueden ser el alcohol o el cigarrillo. Esto también se demuestra en estudios previos donde relacionan factores externos del medio ambiente que intervienen directamente sobre los genes que están implicados en el labio y paladar hendido.

Cuando se evalúa el cigarrillo y el alcohol como factor de riesgo para tener hijos con labio y paladar hendido no sindrómico, no se encuentra una relación estadísticamente significativa, pero si se encuentra una asociación positiva ya que 27 de las madres encuestadas (35\%) presentaron antecedentes como fumadoras con una edad promedio de inicio del hábito a los $16 \pm 2.2$. Veintidós madres (28\%) reportaron haber fumado durante los tres primeros meses de embarazo con un promedio de cigarrillos de $6 \pm 3.5$ diarios. Estas llevaban fumando un pro- 
medio de $11.3 \pm 9.6$ años. Al establecer los factores de riesgo se encontró que variables como antecedentes de fumadora presento un (OR: 1.06 95\% CI: $0.91-12.26$ ) y la edad de inicio de fumar (OR: 1.01 95\% CI: $0.8684-1.12$.).

Cuando se pregunto sobre el consumo de alcohol se encontró que 34 madres (43\%) reportaron estar acostumbradas a tomar bebidas alcohólicas frecuentemente y 16 de las 79 madres encuestadas (20\%) tomaron alcohol durante los tres primeros meses de embarazo. Al establecer factores de riesgo de la cantidad de alcohol consumido durante los tres primeros meses de embarazo y presencia de fisuras orales se encontró un (OR: 1.5 95\% CI: 1.33-17.6.).

Al comparar los resultados de consumo de cigarrillo con presencia de hendiduras orales con los resultados de otros estudios, se encontró que los análisis sugieren una pequeña asociación estadística significativa entre fumar cigarrillo durante el primer trimestre de embarazo y el incremento del riesgo de tener niños con hendiduras. Los resultados del metaanálisis realizado por Wyszynski (1997) sugieren un factor de riesgo promedio entre los 11 estudios revisados con criterios satisfactorios para la hendidura labio-palatina de (OR:1.32 95\% CI:1.10-1.62) que indica un pequeño incremento de riesgo a tener niños con hendidura. ${ }^{22}$ Esta asociación se incrementa al aumentar el número de cigarrillos por día como se observa en el estudio de Shaw y colaboradores, 1991, donde se incrementaba el riesgo cuando la madre fumaba de 1-9 cigarrillos 0 más de 20 cigarrillos. ${ }^{19}$

El consumo de alcohol en la madre embarazada ha mostrado su potencial teratogénico. Estudios de laboratorio con ratones con síndrome de alcoholismo fetal han mostrado una forma leve de holoprosencefalia, deficiencia en la línea media del plato neural anterior que ocasiona que las placodas olfatorias tengan una alteración y cambios secundarios como fisuras orales. Cultivos de neuronas de estos ratones con síndrome de alcoholismo fetal han mostrado alteraciones estructurales y funcionales de astrocitos que ocasionan disturbios en moléculas receptoras de matriz extracelular que están relacionadas con la migración de las células de la cresta neural. ${ }^{23}$

Los datos del presente estudio muestran que un $20 \%$ de las madres consumieron alcohol durante el embarazo, y aunque no se haya una relación de riesgo estadísticamente significativa sí se encuentra una asociación positiva que coincide con los estudios publicados. Munger y
Rommitti (1996) en un estudio de casos y controles muestran como aumenta el riesgo a medida que se incrementa el consumo, encontrando además mayor riesgo para labio y paladar que para paladar.

El presente estudio, también, mostró riesgo con relación a cantidad de alcohol ingerido (OR: 1.5 95\% CI: 0.133-1766). Gary y Lammer (1999) mostraron que las madres que reportaron más de cinco tragos por ocasión, comparadas con las que no tomaron, sí mostraron un incremento en el riesgo de tener niños con labio hendido. Se concluyó que un alto consumo, incrementa los riesgos de la hendidura y en especial del labio hendido. ${ }^{15}$ Lorente Cordier encontró un riesgo aumentado de paladar hendido con el consumo de alcohol (OR:2.28 95\% CI:1.07-3.04) y estableció dos categorías para el consumo de alcohol: más de 70 gramos y menos de 70 gramos. ${ }^{16} \mathrm{~A}$ medida que aumenta la dosis aumenta el riesgo.

Es necesario tener en cuenta que aunque las variables ambientales, como el riesgo y el cigarrillo, pueden aumentar el riesgo de las hendiduras, no son los únicos factores a tener en cuenta. La etiología es poligénica y multifactorial y estos factores ambientales podrían modular la respuesta genética. Se postula que la interacción del cigarrillo con una variante alélica del TGFB3 y del alcohol con el MSX1 son factores importantes asociados con el desarrollo del labio y paladar hendido. Romitti y Cidral examinaron las variantes alélicas de tres genes: (TGFA), (TGFB3) Y (MSX1) y su relación con la exposición durante el embarazo al cigarrillo y al alcohol. Se encontró riesgo asociando más de 10 cigarrillos al día con paladar hendido y variantes alélicas TGFB3 o MSX1. En comparación con el riesgo de consumo de más de cuatro tragos por mes para labio y paladar y variante alélica del MSX1. Los autores sugieren que el desarrollo del labio y paladar hendido puede ser influenciado independientemente por la exposición materna, pero más significativamente por la interacción de exposición a tales factores y variantes alelicas especificas. $^{24}$

Es importante complementar este trabajo con datos de estudios genéticos para un mejor entendimiento de la información. Los reportes en labio y paladar e hipodoncia muestran una influencia genética en su etiología. Posteriores estudios con diseños de casos y controles y mayor tamaño de la muestra aplicada a nuestra población permitirá continuar esta línea de investigación. 


\section{BIBLIOGRAFÍA}

1. McNeil CK. Congenital oral deformities. Br Dent J 1956; 101: 191 -198 .

2. Coccaro PJ. Orthodontics in cleft palate children. Cleft Palate J 1969; 6: $495-505$.

3. Stricker M, Coiné C, Chassagne JF, Simon E, Stricker C, Chassagner S, Fyad JP. Orthopedic treatment of labio-maxillo-palatal clefts: our approach. Rev Stomatol Chir Maxillofac 2001; 102: 190 - 200.

4. McDonal R, Avery D. Odontología pediátrica y del adolescente. 6ta. Edición, Mosby/Doyma Libros, Madrid, 1995.

5. Pruzansky A. The multidiscipline approach of the treatment of cleft palate. Cleft Palate J 1990; 10: 99 -104.

6. Bishara SE, de Arrendondo RS, Vales HP, Jakobsen JR. Dentofacial relationships in persons with unoperated clefts: comparisons between three cleft types. Am J Orthod 1985; 87: $481-507$.

7. Proffit W. Ortodoncia teórica y práctica. Mosby, 1994. Pg. 250-260.

8. Departamento de salud oral de la Misericordia, Universitario Pediátrico. Atención interdisciplinaria en labio y/o paladar fisurado. En: Hernández GA, Bonilla AO, Torres MH. Guías de manejo en estomatología pediátrica. Santa Fe de Bogotá: ECOE ediciones; 1998. p. 227 -229 .

9. Harina FN. Dental development in cleft and noncleft subjects. Angle Orthod 1976; 46: 47 - 50.

10. Susami T, Kuroda T, Amagasa T. Orthodontic treatment of a cleft palate with surgically assisted rapid maxillary expansion. Cleft Palate Craniofac J 1996; 33: 445 - 449.

11. Swennen G, Colle F, De May A. Malevez C. Maxillary distraction in cleft lip palate patients: a review of six cases. J Craniofac Surg 1999; 10: $117-122$.

12. Ross RB. Treatment variables affecting facial growth in complete unilateral cleft lip and palate. Cleft Palate J 1987; 24: 5 - 77.

13. Turvey TA, Vig K, Moriarty J, Hoke J. Delayed bone grafting in the cleft maxilla and palate: a retrospective multidisciplinary analysis. Am J Orthod 1984; 86: $244-256$.

14. Jacobson BN, Rosenstein SW. Early maxillary orthopedics for the newborn cleft lip and palate patient. An impression and an appliance. Angle Orthod 1984; 54: $247-263$.

15. Suzuki A, Takahama Y. A jointed fan-type expander: a newly designed expansion appliance for the upper dental arch of patients with cleft lip and/or palate. Cleft Palate J 1989; 26: 239 - 241.

16. Espinosa H. Ortopedia en pacientes con labio y paladar fisurado. Revista de la Facultad de Odontología de la Universidad de Antioquia 1993.
17. Millard DR Jr, Berkowitz S, Latham RA, Wolfe SA. A discusión of presurgical orthodontics in patients with clefts. Cleft Palate J 1988; 25: $403-412$.

18. Cartagena R. Importancia del tratamiento integral y secuencial de pacientes con labio y paladar hendido. Revista FOC 1988; 38: 37 - 45.

19. Dahllof G, Ussisoo-Joandi R, Ideberg M, Modeer T. Caries, gingivitis, and dental abnormalities in preschool children with cleft lip and/or palate. Cleft Palate J 1989; 26: 233 - 237.

20. Semb. A multidisciplinary management of cleft lip and palate in Oslo, Norway. Philadelphia, 1990.

21. Molina N, Castro I. Factores sociodemograficos en pacientes con labio y/o paladar hendido. [Tesis de Especialización]. Bucaramanga: Universidad Santo Tomás; 2004.

22. Torres EA, Pinzón S. Cigarrillo y alcohol como factores de riesgo en madres para tener hijos con hendidura labio y/o palatina. [Tesis de Especialización]. Bucaramanga: Universidad Santo Tomás; 2004.

23. Wyszynski DF, Wu T. Use of US birth certificate data to estimate the risk of maternal cigarette smoking for oral clefting. Cleft palate Craniofac J 2002; 39: 188 - 192.

24. Sirpa A. Phenotypic and genotypic features of familial hypodontia. Academic Dissertation 2001. Helsinki University.

25. Thesleff I. Homeobox genes and growth factors in regulation of craniofacial and tooth morphogenesis. Acta Odontol Scand 1995; 53: $129-134$.

26. Johnston MC, Bronsky PT. Prenatal craniofacial development: New insights on normal and abnormal mechanisms. Crit Rev Oral Biol Med 1995; 6: 25 - 79. 